\title{
Results from the OPERA experiment
}

\author{
A. Di Crescenzo ${ }^{1,2, a}$ on behalf of the OPERA Collaboration \\ ${ }^{1}$ Dipartimento di Fisica dell'Università Federico II di Napoli \\ ${ }^{2}$ INFN Sezione di Napoli
}

\begin{abstract}
The OPERA neutrino experiment was designed to perform a unique $v_{\tau}$ appearance measurement in the $v_{\mu}$ CNGS beam to confirm the oscillation mechanism in the atmospheric sector $v_{\mu} \rightarrow v_{\tau}$. The detection of $\tau$ leptons produced in $v_{\tau} \mathrm{CC}$ interactions and of their decays is accomplished exploiting the high spatial resolution of nuclear emulsions. Five $v_{\tau}$ candidate events have been detected in the full data sample from 2008-2012 CNGS runs, with an expected background of 0.25 events. The background only hypothesis is rejected with a significance larger than $5 \sigma$. The analysis of the tau neutrino sample in the framework of the $3+1$ neutrino model is also presented. Furthermore OPERA good capabilities in detecting electron neutrino interactions allow setting limits on the $v_{\mu} \rightarrow v_{e}$ oscillation channel.
\end{abstract}

\section{The OPERA detector}

The aim of the OPERA (Oscillation Project with Emulsion-tRacking Apparatus) experiment [1, 2] at the Gran Sasso underground Laboratory (LNGS) was to perform the first direct detection in the appearance mode of $v_{\mu} \rightarrow v_{\tau}$ oscillations. The direct appearance search was based on the detection of $\tau$ leptons produced in charged current interactions (CC) of $v_{\tau}$. The CERN neutrino beam is an almost pure $v_{\mu}$ beam produced by protons accelerated in the CERN SPS and injected in the CNGS beam line. The OPERA experiment $[3,4]$ was installed in the LNGS Hall C.

OPERA was a hybrid apparatus with a modular structure. The detector concept was based on the Emulsion Cloud Chamber (ECC) technique, combined with real-time detection techniques (electronic detectors). The ECC basic unit in OPERA is a brick made of 56 lead plates, providing the required mass, interleaved with 57 nuclear emulsion films, providing the necessary spatial and angular resolution. The electronic detectors were used to trigger the neutrino interactions, to locate the brick in which the interaction took place, to identify muons and measure their momentum and charge. The analysis of nuclear emulsion films is performed in the OPERA scanning laboratories in Europe and Japan by fast automatic scanning systems developed to reconstruct with sub-micrometric accuracy the neutrino interaction events.

The detector shown in Fig. 1 is composed of two identical supermodules (SM1 and SM2), each consisting of a target section made of arrays of bricks coupled to a scintillator Target Tracker detector (TT) to trigger the read-out and localize neutrino interactions within the target. Each target section is followed by a muon spectrometer, namely a dipole magnet instrumented with resistive plate chambers

ae-mail: dicrescenzo@na.infn.it 
(RPCs) and drift tubes detectors (PT), to measure the muon charge and momentum.

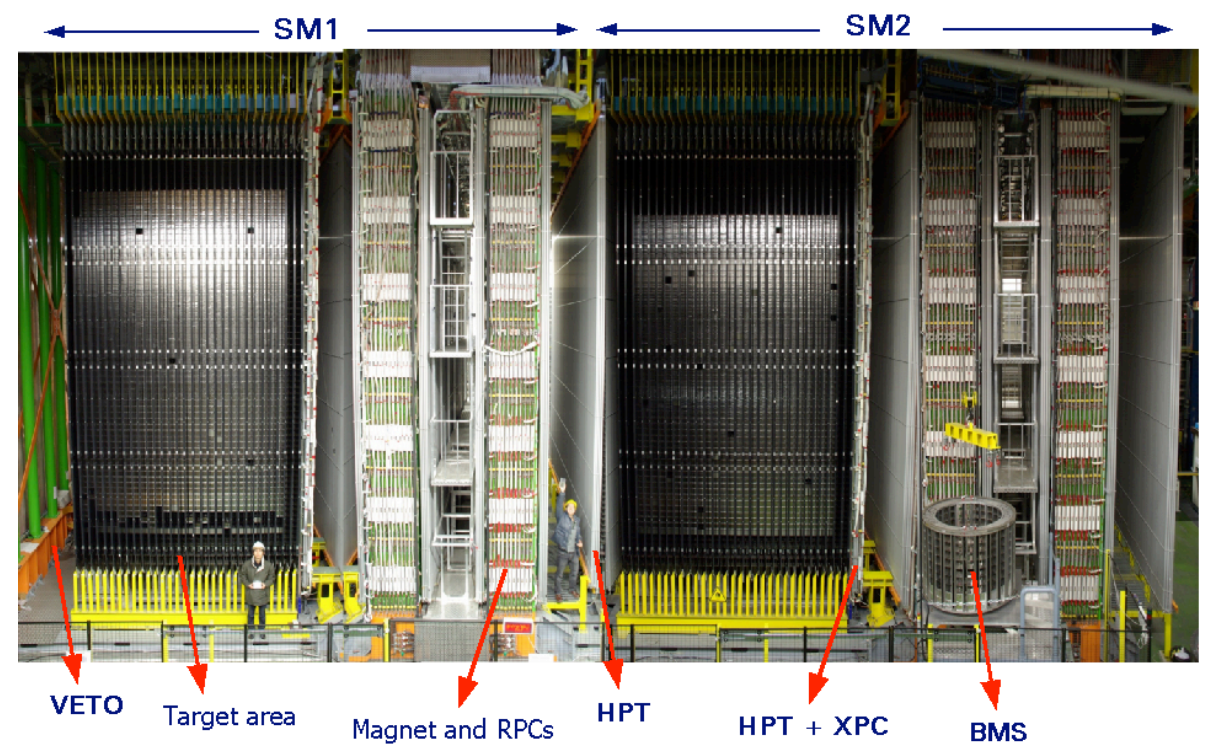

Figure 1. View of the OPERA detector. The upper horizontal lines indicate the position of the two identical supermodules (SM1 and SM2). Arrows show the position of a target area (ECC brick walls interleaved with planes of plastic scintillators), the VETO planes, drift tube detector (HPT) planes, the magnet and the RPC. A part of the Brick Manipulator System (BMS) is also visible.

\section{Event reconstruction and analysis}

The electronic detector data are used to identify the brick in which the neutrino interaction occurred. Events are classified as Charged Current like $(1 \mu)$ if a track is tagged as a muon or if a minimum number of detector walls is traversed, or as Neutral Current like $(0 \mu)$ in the complementary case. The bricks tagged as containing a neutrino interaction vertex were extracted from the detector, their emulsion films developed and sent to the scanning laboratories in Europe and Japan, where a search for $\tau$ decays is performed using custom automated optical microscopes.

The search for the neutrino interaction vertex starts from a set of tracks predicted using information from the electronic detectors. Confirmed tracks are followed upstream in the brick from the most downstream emulsion film to the possible interaction vertex. A volume of $1 \mathrm{~cm}^{2}$ by 5 films upstream and 10 films downstream is scanned in order to fully explore the vertex area and possibly find other associated particle tracks. A procedure called decay search is then applied [5] in order to search for charged or neutral decay topologies, secondary interactions or gamma-ray conversions. If a secondary vertex is found, a full kinematical analysis is performed combining the measurements in the nuclear emulsion with data from the electronic detectors. The momentum of charged particles can be measured in emulsions by the Multiple Coulomb Scattering up to $6 \mathrm{GeV} / \mathrm{c}$ with resolution better than $22 \%$ 
using the angular deviations [6]; it can be measured up to $12 \mathrm{GeV} / \mathrm{c}$ with a resolution better than $33 \%$ using position deviations. For muons crossing the spectrometers, the momentum is measured with a resolution better than $22 \%$ up to $30 \mathrm{GeV} / \mathrm{c}$. The muon charge is also determined [7]. The hint of a decay topology is the observation of an impact parameter, defined as the minimum distance between the track and the reconstructed vertex, excluding low momentum tracks, greater than $10 \mu \mathrm{m}$.

\section{Search for $v_{\mu} \rightarrow v_{\tau}$ oscillations}

In the analysed sample, five $v_{\tau}$ candidates were observed. The first $v_{\tau}$ candidate, observed in the 2008-2009 data sample [8], has seven prongs at the primary vertex. One track exhibits a kink topology; the daughter track is identified as a hadron by its interaction. In addition, two $\gamma$-rays point to the secondary vertex. Their invariant mass is $120 \pm 20$ (stat. $) \pm 35$ (syst.) $\mathrm{MeV} / \mathrm{c}^{2}$, compatible with the $\pi^{0}$ mass. Its combination with the secondary hadron, assumed to be a $\pi^{-}$, gives an invariant mass of $640_{-80}^{+125}(\text { stat. })_{-90}^{+100}$ (syst. $) \mathrm{MeV} / \mathrm{c}^{2}$. The decay mode is therefore compatible with $\tau \rightarrow \rho(770) v_{\tau}$, whose branching ratio is about $25 \%$.

The second $v_{\tau}$ candidate event, found in the 2011 data sample [9], is a 2-prong neutrino interaction with a $1.54 \mathrm{~mm}$ track (associated with $\tau$ lepton) and a longer track identified as a hadron being incompatible with a muon track because of its range. The $\tau$ lepton exhibits a 3-prong decay topology. The daughter particles are identified as hadrons on the basis of momentum-range consistency. The kinematical analysis of the event satisfies all the specified criteria for the $\tau \rightarrow 3 h$ decay channel.

The third $\nu_{\tau}$ candidate was observed in the $20121 \mu$ data sample [10]. The primary vertex is given by two tracks and a $\gamma$-ray. The first track is associated to a hadron from the momentum-range correlation. The other track from the $\tau$ lepton decaying into a muon. The muon momentum determined from the track reconstructed by the electronic detectors is $2.8 \pm 0.2 \mathrm{GeV} / \mathrm{c}$; its negative charge is assessed with $5.6 \sigma$ significance. All the kinematical criteria for the selection of $\tau \rightarrow \mu$ decays are satisfied.

The fourth $v_{\tau}$ event was observed in $20120 \mu$ data sample. The primary vertex is defined by four tracks and two $\gamma$-rays. One track exhibits a kink topology. The daughter track exits the target, stopping in the spectrometer after crossing three RPC planes. Momentum-range correlation allows to identify it as a hadron.

The fifth $v_{\tau}$ candidate was found in $20120 \mu$ sample [11]. The primary vertex consists of the $\tau$ candidate track, since it exhibits a kink topology, and a charged particle track ( $p_{1}$, see Fig. 2$)$. In addition, four forward-going and two backward-going nuclear fragments are observed, all pointing to the primary vertex. The $\tau$ candidate decays, after a flight length of $960 \pm 30 \mu \mathrm{m}$, into one charged particle identified as a hadron since a secondary interaction is reconstructed 22 lead plates downstream yielding four charged particles and a photon. Figure 2 shows the display of the event as reconstructed in the brick. The momentum of the charged particle producing the primary track $p_{1}$ is $1.0 \pm 0.1 \mathrm{GeV} / \mathrm{c}$. The particle is identified as a hadron from its interaction in the downstream brick. This, together with the null result from the search for large angle tracks, allows to rule out the presence of a muon at the primary vertex. The kinematical analysis of the event satisfies all the specified criteria for the $\tau \rightarrow h$ decay channel.

In the analysed data sample the expected number of background events is $0.04,0.17,0.004$ and 0.03 in the $\tau \rightarrow h, \tau \rightarrow 3 h, \tau \rightarrow \mu$ and $\tau \rightarrow e$ channels, respectively, mainly from charmed events with an undetected primary muon, hadronic re-interactions (for the hadronic decay channels) and large angle muon scattering (for the $\tau \rightarrow \mu$ channel). From the observation of the five candidate events and taking into account the signal-to-noise ratio for each decay channel, non-null observation can be assessed with 5.1 sigma significance. 


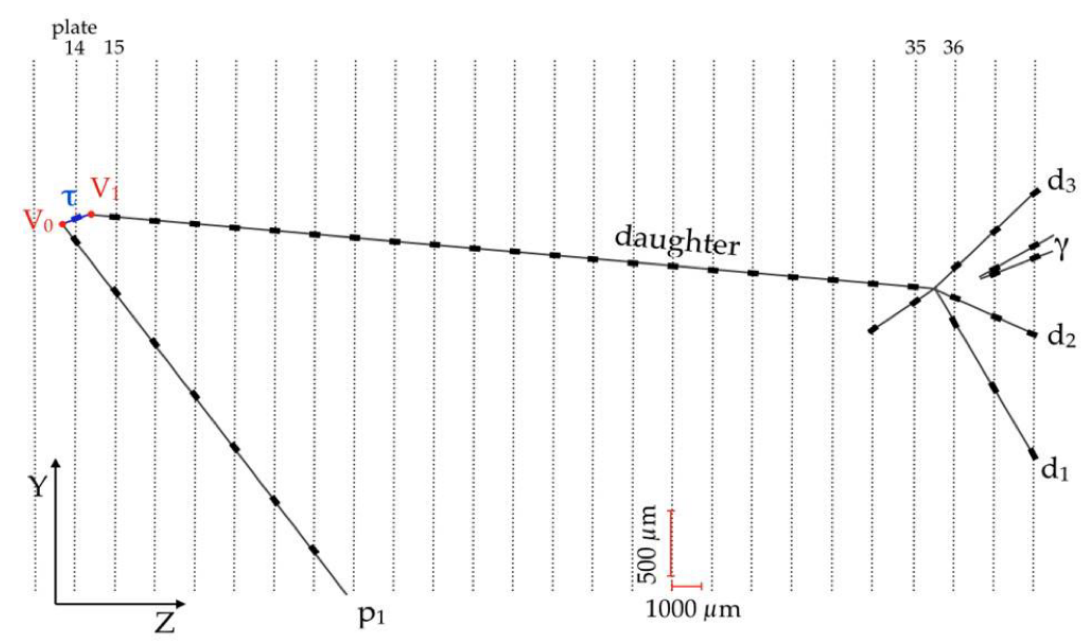

Figure 2. Event display of the fifth $v_{\tau}$ candidate event in the horizontal projection longitudinal to the neutrino direction. The event primary and secondary vertices are indicated as $\mathrm{V}_{0}$ and $\mathrm{V}_{1}$, respectively.

\section{Search for sterile neutrinos}

The OPERA experiment can test existence of a fourth (sterile) neutrino state at the eV mass scale by looking for deviations from the standard three-neutrino model predictions. In presence of a fourth sterile neutrino with mass $m_{4}$, the oscillation probability is a function of the $4 \times 4$ mixing matrix $U$ and of the three squared mass differences. In the framework of the $3+1$ model, at values of $\Delta \mathrm{m}_{14}^{2}>$ $1 \mathrm{eV}^{2}$, the measured $90 \%$ C.L. upper limit on the mixing term $\sin 2 \theta_{\mu \tau}=2\left|U_{\mu 4} \| U_{\tau 4}\right|$ is 0.116 , independently of the mass hierarchy of the three standard neutrinos. The OPERA experiment extends the exclusion limits on $\Delta \mathrm{m}_{14}^{2}$ in the $v_{\mu} \rightarrow v_{\tau}$ appearance channel down to values of $10^{-2} \mathrm{eV}^{2}$ at large mixing for $\sin ^{2} 2 \theta_{\mu_{\tau}} \geq 0.5$, as shown in Fig. 3 .

A systematic search for $v_{e}$ events was applied to $5050 \mu$ events located in 2008 and 2009 data sample [16]. The number of observed $v_{e}$ interactions (19 events) is compatible with that expected from the $v_{e}$ beam contamination $(19.8 \pm 2.8)$. In Fig. 4 the reconstructed energy distribution of the detected candidates is compared with the energy spectra of the $v_{e}$ beam contamination, the expected oscillated $v_{e}$ from the three-flavour oscillation and the background.

The observation is compatible with the non-oscillation hypothesis; an upper limit $\sin ^{2}\left(2 \theta_{13}\right)<0.44$ is derived at the $90 \%$ C. L..

In the non-standard oscillation framework and in the one mass scale dominance approximation (2 neutrino mixing) the exclusion plot in Fig. 5 was derived. A complete analysis in the $3+1$ scheme based on the statistics of the full data sample (factor of 2.5 increase) is ongoing. 


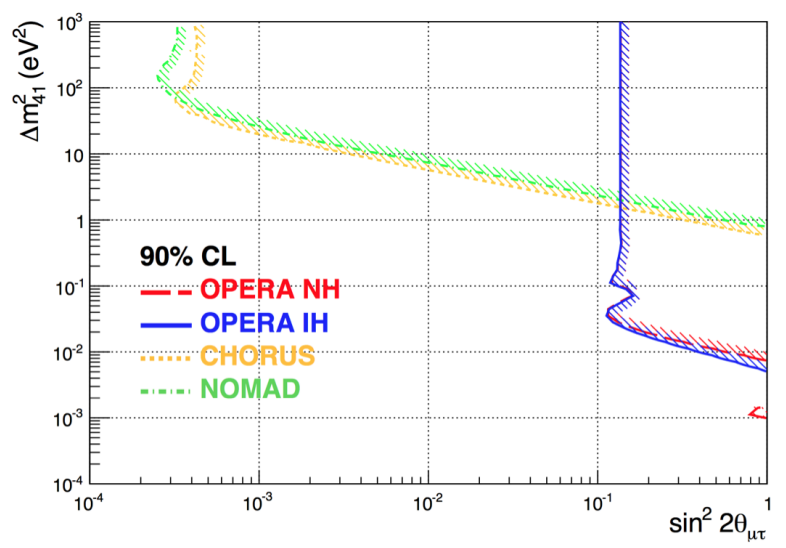

Figure 3. OPERA preliminary $90 \%$ C.L. exclusion limits in the $\Delta \mathrm{m}_{41}^{2} \mathrm{vs} \sin ^{2} 2 \theta_{\mu \tau}$ parameter space for the normal ( $\mathrm{NH}$, red) and inverted ( $\mathrm{IH}$, blue) hierarchy of the three standard neutrino masses. The exclusion plots by NOMAD [14] and CHORUS [15] are also shown. Shaded bands are drawn to indicate the excluded regions.

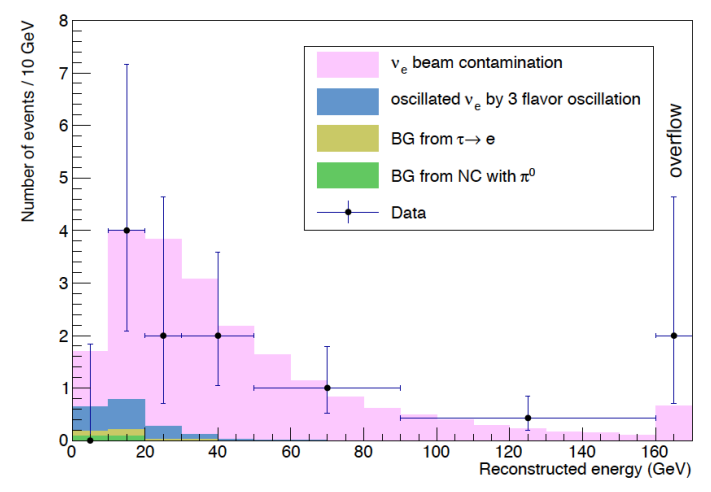

Figure 4. Energy distribution of the detected $v_{e}$ events comparing to the expectations of beam $v_{e}$ and oscillated $v_{e}$.

\section{References}

[1] M. Guler et al. (OPERA Collab.), CERN-SPSC-97-24, 1997.

[2] M. Guler et al. (OPERA Collab.), CERN-SPSC-2001-25, 2001.

[3] R. Acquafredda et al. (OPERA Collab.), JINST 4 (2009) P04018.

[4] R. Acquafredda et al. (OPERA Collab.), New J. Phys. 8 (2006) 303.

[5] N. Agafanova et al. (OPERA Collab.), Eur. Phys. J C 89 (2014) 2986.

[6] N. Agafanova et al. (OPERA Collab.), New J. Phys. 14 (2012) 013026.

[7] N. Agafanova et al. (OPERA Collab.), New J. Phys. 13 (2011) 053051.

[8] N. Agafanova et al. (OPERA Collab.), Phys. Lett. B 691 (2010) 138.

[9] N. Agafanova et al. (OPERA Collab.), JHEP 11 (2013) 036. 


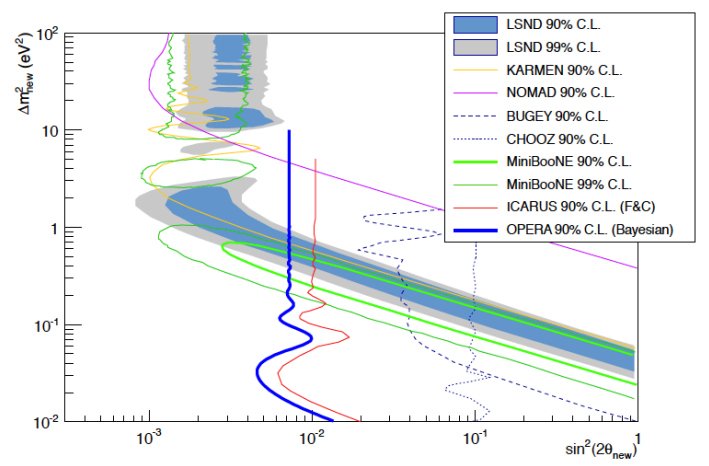

Figure 5. The exclusion plot for the parameters of the non-standard $v_{\mu} \rightarrow v_{e}$ oscillation, obtained from this analysis using the Bayesian method, is shown. Limits from other experiments are also shown.

[10] N. Agafanova et al. (OPERA Collab.), Phys. Rev. D 89 (2014) 051102.

[11] N. Agafanova et al. (OPERA Collab.), Prog. Theor. Exp. Phys. (2014) 101C01.

[12] A. Aguilar-Arevalo et al. (LSND Collaboration), Phys. Rev. D 64 (2001) 112007;

A. A. Aguilar-Arevalo et al. (MiniBooNE Collaboration), Phys. Rev. Lett. 110 (2013) 161801;

G. Mention et al., Phys. Rev. D 83 (2011) 073006;

Th. A. Muller et al., Phys. Rev. C 83 (2011) 054615;

C. Giunti and M. Laveder, Phys. Rev. C 83 (2011) 065504.

[13] N. Agafonova et al. (OPERA Collab.) JHEP 1506 (2015) 069.

[14] P. Astier et al. (NOMAD Collaboration), Nucl. Phys. B 6113 (2001).

[15] E. Eskut et al. (CHORUS Collaboration), Nucl. Phys. B 793326 (2008).

[16] N. Agafanova et al. OPERA Collab.) JHEP 07(2013) 004. 